\title{
Control of Glyphosate and Acetolactate Synthase Resistant Common Ragweed (Ambrosia artemisiifolia L.) in Soybean (Glycine max L.) with Preplant Herbicides
}

\author{
Annemarie C. Van Wely ${ }^{1}$, Nader Soltani' ${ }^{1}$, Darren E. Robinson'1, David C. Hooker1, \\ Mark B. Lawton' ${ }^{2}$, Peter H. Sikkema1 \\ ${ }^{1}$ University of Guelph (Ridgetown Campus), Ridgetown, Canada \\ ${ }^{2}$ Monsanto Canada, Guelph, Canada \\ Email: soltanin@uoguelph.ca
}

Received 6 November 2014; revised 5 December 2014; accepted 13 December 2014

Copyright $@ 2014$ by authors and Scientific Research Publishing Inc.

This work is licensed under the Creative Commons Attribution International License (CC BY). http://creativecommons.org/licenses/by/4.0/

(c) (i) Open Access

\section{Abstract}

A population of common ragweed in Ontario was confirmed to be resistant to glyphosate in 2011. Group 2 [acetolactate synthase (ALS) inhibitors] resistant common ragweed was first confirmed in Ontario in 2000. Previously, glyphosate provided excellent control of common ragweed in glyphosate resistant soybean but with the confirmation of glyphosate resistant (GR) common ragweed, alternative herbicides need to be evaluated. Eight field trials with preplant herbicides were completed over two years (2013 and 2014) in fields with confirmed GR common ragweed. Tankmixes of glyphosate and linuron or metribuzin provided $88 \%-99 \%$ and $86 \%-98 \%$ control 4 weeks after application (WAA) and $80 \%-92 \%$ and $80 \%$ - $95 \%$ control 8 WAA, respectively. However, these herbicides also had among the highest environmental impact of the herbicides tested. Based on the results of these studies, GR common ragweed can be controlled with residual herbicides when applied preemergence in soybean. Currently, there are no post emergence herbicides that provide adequate control of GR common ragweed, therefore, preemergence herbicides with residual are essential for full season control.

\section{Keywords}

Glyphosate Resistance, Multiple Herbicide-Resistant Weeds, Preplant Herbicides, Preemergence Herbicides 


\section{Introduction}

Soybean (Glycine max L. Merr.) is an important crop in Ontario with approximately 800,000 hectares grown annually [1]. Soybean is used for livestock feed and human consumption in the form of oil and specialty food [1]. Soybean is seeded from late April until early June. This timing coincides with the emergence of many summer annual weeds, including common ragweed. Generally, these weeds can be controlled with a preplant or post emergence herbicide application, with glyphosate being the most widely used.

Common ragweed (Ambrosia artemisiifolia L.) is the fifth most common annual weed in southern Ontario [2]. Common ragweed is a competitive weed in soybean, resulting in yield losses of $132 \mathrm{~kg} \cdot \mathrm{ha}^{-1}$ when there are only four plants per meter of row [3]. It is also a concern for allergy sufferers as it is one of the main causes of hay fever [4], and is listed as a noxious weed under the Ontario Weeds Act [5]. Due to its widespread distribution, competitiveness and classification as a noxious weed, landowners must eliminate common ragweed from their properties.

The first glyphosate resistant (GR) population of common ragweed was found in Missouri in 2004 [6] [7]. GR common ragweed is now confirmed in eight US states and one Canadian province (Heap 2013). Pollard (2007) reported that glyphosate $\left(1.68 \mathrm{~kg} \cdot \mathrm{ha}^{-1}\right)$, glyphosate $\left(0.84 \mathrm{~kg} \cdot \mathrm{ha}^{-1}\right)$ plus chlorimuron $\left(0.013 \mathrm{~kg} \cdot \mathrm{ha}^{-1}\right) \mathrm{and}_{\mathrm{gly}-}$ phosate $\left(0.84 \mathrm{~kg} \cdot \mathrm{ha}^{-1}\right)$ plus imazethapyr $\left(0.071 \mathrm{~kg} \cdot \mathrm{ha}^{-1}\right)$ provided greater than $80 \%$ control of the GR biotype in Missouri. Greater control was obtained when herbicides were tankmixed with the label rate of glyphosate compared to when they were applied alone [6].

In 2011, GR common ragweed was confirmed in Ontario in a soybean field that had inadequate control following the application of glyphosate [7]. In addition, in 2000, common ragweed was found to be resistant to Group 2 (ALS inhibitors) herbicides [7]. The GR common ragweed biotypes in Ontario are resistant to all three families of Group 2 herbicides (sulfonylureas, imidazolinones and triazolopyrimidines), therefore these biotypes are considered to be multiple resistant (unpublished data). Although previous research has been completed on alternative herbicides for the control of GR common ragweed, this research found that the Group 2 herbicides were the most effective, however, because the Ontario biotype is multiple resistant, these options are not effective. In addition, the herbicides available, the recommend label rates, and the herbicide formulations differ between Canada and the United States of America. Therefore, the objective of this study was to determine the control of multiple resistant common ragweed in soybean in Ontario with preplant herbicides and to document the environmental impact (EI) of these herbicides.

\section{Materials and Methods}

Eight field experiments were conducted over a two-year period (2013 and 2014) in fields with confirmed multiple resistant (Group 2 and 9) common ragweed to determine the efficacy of preplant (PP) herbicides in glyphosate resistant (GR) soybean. Two sets of experiments were conducted; the first was named "enhanced burndown" which included herbicides with limited residual activity while the second experiment, named "burndown plus residual” contained herbicides that provide residual weed control. In 2013, both experiments were conducted on one site near Windsor, Ontario (S1, S2), because this was the only known site with confirmed multiple resistant common ragweed in Ontario. The experiments were separated in time and sprayed 12 days apart. An additional site was confirmed later in 2013, so in 2014, the enhanced burndown experiments were conducted on two sites S3 (Windsor) and S4 (Belle River), while both the burndown plus residual experiments were conducted on the Windsor site (S3, S4), and sprayed three days apart.

The experiments were established as a randomized complete block design with 3 replications at the Windsor site and 4 replications at the Belle River site due to space available. The plots were $2.25 \mathrm{~m}$ wide (3 rows of soybean spaced $0.75 \mathrm{~m}$ apart) and $6 \mathrm{~m}$ in length at the Windsor site and $3.0 \mathrm{~m}$ wide (4 rows of soybean spaced 0.75 $\mathrm{m}$ apart) and $8 \mathrm{~m}$ in length at the Belle River site. Herbicides were applied prior to soybean seeding using a $\mathrm{CO}_{2}$ pressurized backpack sprayer, which was calibrated to deliver $200 \mathrm{~L} \cdot \mathrm{ha}^{-1}$ of liquid at $210 \mathrm{kPa}$. The boom used at the Windsor site was $1 \mathrm{~m}$ wide with three ULD 120 - 20 nozzles (Hypro, New Brighton, MN) spaced $50 \mathrm{~cm}$ apart, while the boom used at Belle River was $1.5 \mathrm{~m}$ with four nozzles. Both experiments included a weedy and a weed free control. The weed free control was maintained weed free with glyphosate (1800 g a.e. ha ${ }^{-1}$ ), s-metolachlor (1600 g a.i. $\mathrm{ha}^{-1}$ ) and metribuzin (653 $\mathrm{g}^{\text {a.i. }} \mathrm{ha}^{-1}$ ) applied preplant followed by hand hoeing as required. Location, soil properties, seeding date, herbicide application date and common ragweed height and density at the time of application are listed in Table 1. The herbicides evaluated in the enhanced burndown experiment 
Table 1. Location, agronomic information and height and density of multiple resistant (Group 2 and 9) common ragweed for experiments in Ontario, Canada in 2013 and 2014.

\begin{tabular}{|c|c|c|c|c|c|c|c|c|c|}
\hline \multicolumn{10}{|c|}{ Enhanced burndown } \\
\hline \multirow[b]{2}{*}{ Location } & \multirow[b]{2}{*}{ Year } & \multirow[b]{2}{*}{ Closest city } & \multicolumn{3}{|c|}{ Soil characteristics } & \multirow{2}{*}{$\begin{array}{c}\text { Seeding } \\
\text { Date }\end{array}$} & \multirow{2}{*}{$\begin{array}{c}\begin{array}{c}\text { Herbicide } \\
\text { application }\end{array} \\
\text { Date } \\
\end{array}$} & \multicolumn{2}{|c|}{ Common ragweed } \\
\hline & & & Texture & OM (\%) & pH & & & Size $(\mathbf{c m})$ & Density $\left(\mathrm{m}^{-2}\right)$ \\
\hline $\mathrm{S} 1$ & 2013 & Windsor & Clay & 3.1 & 7.2 & May-27 & May-15 & up to 5 & 157 \\
\hline S2 & 2013 & Windsor & Clay & 3.1 & 7.2 & May-27 & May-27 & up to 10 & 101 \\
\hline S3 & 2014 & Windsor & Clay Loam & 3.4 & 7.2 & May-26 & May-22 & up to 9 & 1925 \\
\hline S4 & 2014 & Belle River & Clay Loam & 2.8 & 7.5 & May-26 & May-25 & up to 4 & 64 \\
\hline \multicolumn{10}{|c|}{ Burndown plus residual } \\
\hline S1 & 2013 & Windsor & Clay & 3.1 & 7.2 & May-27 & May-15 & up to 5 & 327 \\
\hline S2 & 2013 & Windsor & Clay & 3.1 & 7.2 & May-27 & May-27 & up to 10 & 45 \\
\hline S3 & 2014 & Windsor & Clay Loam & 3.4 & 7.2 & May-26 & May-22 & up to 5 & 1522 \\
\hline S4 & 2014 & Windsor & Clay Loam & 3.4 & 7.2 & May-26 & May-25 & up to 10 & 1061 \\
\hline
\end{tabular}

are listed in Tables 2-4 and the herbicides evaluated in the burndown plus residual experiment are listed in Tables 5-8. The herbicide rates used are the maximum labeled rates registered for use in Ontario.

Soybean injury was assessed 2 and 4 weeks after application (WAA). Injury was rated on a scale of $0 \%$ to $100 \%$, where 0 was no injury and 100 was plant death. Common ragweed control ratings were completed 4 and 8 WAA on a scale of $0 \%$ (no control) to $100 \%$ (plant death). Common ragweed density and dry weight were determined 4 WAA in the enhanced burndown experiment and 8 WAA in the burndown plus residual experiment by counting the common ragweed plants in two $0.25 \mathrm{~m}^{2}$ quadrats in each plot and then cutting the plants at the soil surface. The plants were then bagged, dried at $60^{\circ} \mathrm{C}$ to a constant moisture and weighed. At maturity, soybean from each plot were cut at the soil surface from $2 \mathrm{~m}$ from the center row of each plot and threshed in a stationary threshing machine. The grain weight and moisture content were recorded. Soybean yield is reported as a percent of the weed free control. Yield was not obtained at the Belle River (S4) site due to the interference of other weed species. Yield at S2 is not displayed due to the low population of common ragweed in the trial in 2013.

Data were analyzed using the PROC MIXED procedure in SAS (Ver. 9.2, SAS Institute Inc., Cary, NC). Variances were partitioned into the fixed effect of herbicide treatment and environment (year and location) and the random effects of replication and location by treatment interaction. The $\mathrm{Z}$ test was used to test the significance of the random effects as well as the interaction between the fixed and random effects. The significance of the fixed effects was tested using the F test. Locations were analyzed separately or grouped together to ensure there was no significant environment by treatment interactions. Residuals were plotted to ensure that error terms were homogenous and independent. The Shapiro-Wilk statistic was generated using the UNIVARIATE procedure in SAS to test for normality. When necessary, the highest Shapiro-Wilk statistic generated when transforming the data (natural log, square root or arcsine square root) was applied to the data. Fisher's protected LSD at $\mathrm{P}<0.05$ was used to separate the means.

In the enhanced burn down study, the weed biomass data was log transformed for analysis. All weed control data was analyzed on the untransformed data. For the burndown plus residual study at 4 WAA, S2 data were analyzed after being log transformed, while data from the other sites were untransformed (S1 and S3, S4). All control data at 8 WAA was analyzed on the untransformed scale. The weed biomass data was square root transformed for S1 and S2 and untransformed for S3 and S4.

The environmental impact (EI) of each herbicide combination was calculated by taking the environmental impact quotient (EIQ) of the herbicide as calculated by Kovach et al. and multiplying it by the rate used [8]-[12]. The EI of each herbicide combination is listed in Table 4 for the enhanced burndown and Table 8 for the burndown plus residual experiment.

\section{Results and Discussion}

\subsection{Enhanced Burndown}

Soybean injury at 2 and 4 WAA was minimal (data not shown). The injury observed included leaf speckling and 
Table 2. Percent control of multiple resistant (Group 2 and 9) common ragweed 4 and 8 WAA in the "enhanced burndown” study conducted in Ontario, Canada in 2013 and 2014.

\begin{tabular}{|c|c|c|c|c|c|c|}
\hline & & \multicolumn{3}{|c|}{ Percent control 4 WAA } & \multicolumn{2}{|c|}{ Percent control 8 WAA } \\
\hline \multicolumn{7}{|c|}{ Rate } \\
\hline Treatment & (g a.i/a.e. ha ${ }^{-1}$ ) & $\mathrm{S} 1^{\mathrm{z}}$ & S2 & S3, S4 & S1, S2 & S3, S4 \\
\hline Weedy control & & 0 e & $0 \mathrm{i}$ & $0 \mathrm{~g}$ & $0 \mathrm{~g}$ & $0 \mathrm{f}$ \\
\hline Weed Free control & & $100 \mathrm{a}$ & $100 \mathrm{a}$ & $100 \mathrm{a}$ & $100 \mathrm{a}$ & $100 \mathrm{a}$ \\
\hline Glyphosate & 900 & $20 \mathrm{~d}$ & $7 \mathrm{hi}$ & $48 \mathrm{f}$ & $12 \mathrm{fg}$ & 38 e \\
\hline Glyphosate + 2,4-D & $900+500$ & $83 \mathrm{~b}$ & $75 \mathrm{~cd}$ & $72 \mathrm{~cd}$ & $69 \mathrm{~b}$ & $56 \mathrm{~cd}$ \\
\hline Glyphosate + amitrole & $900+2000$ & $83 \mathrm{~b}$ & 65 de & 81 bc & $63 \mathrm{bc}$ & $59 \mathrm{~cd}$ \\
\hline Glyphosate + carfentrazone & $900+17.5$ & $20 \mathrm{~d}$ & $15 \mathrm{gh}$ & 57 ef & 20 ef & 45 de \\
\hline Glyphosate + chlorimuron & $900+9$ & $38 \mathrm{c}$ & $13 \mathrm{gh}$ & 66 de & 18 ef & 52 cde \\
\hline $\begin{array}{c}\text { Glyphosate + chlorimuron } \\
+ \text { flumioxazin }\end{array}$ & $900+9+71$ & $27 \mathrm{~cd}$ & $53 \mathrm{f}$ & $72 \mathrm{~cd}$ & 38 de & $59 \mathrm{~cd}$ \\
\hline Glyphosate + cloransulam ${ }^{y}$ & $900+17.5$ & $20 \mathrm{~d}$ & $23 \mathrm{~g}$ & 65 de & $17 \mathrm{f}$ & 53 cde \\
\hline Glyphosate + flumioxazin & $900+71$ & $33 \mathrm{~cd}$ & 57 ef & 65 de & $46 \mathrm{~cd}$ & 47 de \\
\hline Glyphosate + glufosinate & $900+500$ & $73 \mathrm{~b}$ & 62 ef & 77 bcd & 58 bc & $65 \mathrm{bc}$ \\
\hline Glyphosate + paraquat & $900+1100$ & $82 \mathrm{~b}$ & 79 bc & 75 bcd & $68 \mathrm{~b}$ & $59 \mathrm{~cd}$ \\
\hline 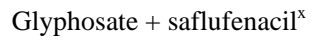 & $900+25$ & $80 \mathrm{~b}$ & $90 \mathrm{ab}$ & $89 a b$ & $69 \mathrm{~b}$ & $76 \mathrm{~b}$ \\
\hline
\end{tabular}

${ }^{\mathrm{z}}$ Abbreviations: S1, S2, S3, Windsor; S4, Belle River; WAA, weeks after application of herbicide. ${ }^{\mathrm{y}}$ Added Agral 90 (0.25\% v/v) and UAN 28\% (2.5\% $\mathrm{v} / \mathrm{v})$. ${ }^{\mathrm{x}}$ Added Merge $(1.0 \% \mathrm{v} / \mathrm{v})$. a - i means followed by the same letter are not significantly different according to Fisher's Protected LSD at P $=0.05$.

Table 3. Multiple resistant (Group 2 and 9) common ragweed biomass 4 WAA and soybean yield in the "enhanced burndown" study conducted in Ontario, Canada in 2013 and $2014^{\mathrm{z}}$.

\begin{tabular}{|c|c|c|c|c|c|}
\hline & \multirow[b]{2}{*}{ Rate } & \multicolumn{3}{|c|}{ Weed biomass 4 WAA } & Soybean yield \\
\hline & & S1,S2 & S3 & S4 & S1, S3 \\
\hline Treatment & (g a.i./a.e ha ${ }^{-1}$ ) & & $\mathrm{g} \cdot \mathrm{m}^{-2}$ & & \% Reduction ${ }^{w}$ \\
\hline Weedy control & & 109.0 a & $120.7 \mathrm{a}$ & 45 a & $73 \mathrm{a}$ \\
\hline Weed free control & & $0 \mathrm{~g}$ & $0 \mathrm{~d}$ & 0 e & $0 \mathrm{~d}$ \\
\hline Glyphosate & 900 & 28.8 bc & 57.7 c & $1.5 \mathrm{c}$ & $70 \mathrm{ab}$ \\
\hline Glyphosate + 2,4-D & $900+500$ & $9.8 \mathrm{~cd}$ & $13.2 \mathrm{~d}$ & $0.66 \mathrm{~cd}$ & $46 \mathrm{abc}$ \\
\hline Glyphosate + amitrole & $900+2000$ & $2.1 \mathrm{ef}$ & $13.8 \mathrm{~d}$ & 0.7 cde & 42 bc \\
\hline Glyphosate + carfentrazone & $900+17.5$ & $40.0 \mathrm{ab}$ & $88.1 \mathrm{~b}$ & $4.5 \mathrm{~b}$ & $74 \mathrm{a}$ \\
\hline Glyphosate + chlorimuron & $900+9$ & 7.0 de & $9.5 \mathrm{~d}$ & $1.2 \mathrm{~cd}$ & $52 \mathrm{abc}$ \\
\hline $\begin{array}{c}\text { Glyphosate + chlorimuron } \\
\text { + flumioxazin }\end{array}$ & $900+9+71$ & $2.1 \mathrm{efg}$ & $8.9 \mathrm{~d}$ & $1.2 \mathrm{~cd}$ & $68 \mathrm{ab}$ \\
\hline Glyphosate + cloransulam ${ }^{y}$ & $900+17.5$ & $0.3 \mathrm{fg}$ & $4.1 \mathrm{~d}$ & 0.9 cde & $74 \mathrm{a}$ \\
\hline Glyphosate + flumioxazin & $900+71$ & $35.2 \mathrm{ab}$ & $72.1 \mathrm{bc}$ & $1.3 \mathrm{c}$ & 74 a \\
\hline Glyphosate + glufosinate & $900+500$ & $39.6 \mathrm{ab}$ & $65.7 \mathrm{bc}$ & 0.1 de & $36 \mathrm{c}$ \\
\hline Glyphosate + paraquat & $900+1100$ & $14.7 \mathrm{bcd}$ & $52.7 \mathrm{c}$ & 0.3 cde & $42 \mathrm{bc}$ \\
\hline Glyphosate + saflufenacil $^{\mathrm{x}}$ & $900+25$ & $23.0 \mathrm{bcd}$ & 71.2 bc & $0.1 \mathrm{de}$ & $24 \mathrm{c}$ \\
\hline
\end{tabular}

${ }^{\mathrm{z}}$ Abbreviations: S1, S2, S3, Windsor; S4, Belle River; WAA, weeks after application of herbicide. ${ }^{\mathrm{y}}$ Added Agral 90 (0.25\% v/v) and UAN 28\% (2.5\% $\mathrm{v} / \mathrm{v}) .{ }^{\mathrm{x}}$ Added Merge $(1.0 \% \mathrm{v} / \mathrm{v})$. ${ }^{\mathrm{w}}$ Compared to the weed free control. a - e means followed by the same letter are not significantly different according to Fisher's Protected LSD at $\mathrm{P}=0.05$.

bronzing which may have been caused by splash burn due to the frequent rain events after herbicide application in both 2013 and 2014.

At 4 WAA, S3 and S4 control data could be combined, while S1 and S2 data had to be analyzed individually. Glyphosate applied alone provided 7\% to $48 \%$ control of GR common ragweed (Table 2). Glyphosate plus 2,4-D, applied preplant provided $72 \%$ to $83 \%$ control of GR common ragweed. Glyphosate plus amitrole provided greater than $80 \%$ control at three of the four sites (S1, S3, S4), however, only provided 65\% control at S2. Glyphosate plus carfentrazone provided $15 \%$ to $57 \%$ control of GR common ragweed, which was the lowest of 
Table 4. Environmental impact of herbicides used in the "enhanced burndown" study conducted in Ontario, Canada in 2013 and $2014^{\mathrm{z}}$.

\begin{tabular}{cccc}
\hline Active ingredient(s) & Individual EIQ values & Product rate & EI \\
\hline Glyphosate & & (g a.i/a.e. ha ${ }^{-1}$ ) & \\
\hline Glyphosate + 2,4-D ester & 15.3 & 900 & 13.8 \\
Glyphosate + amitrole & $15.3+15.33$ & $900+500$ & 21.5 \\
Glyphosate + carfentrazone & $15.3+31.80$ & $900+2000$ & 77.4 \\
Glyphosate + chlorimuron & $15.3+20.18$ & $900+17.5$ & 17.3 \\
Glyphosate + chlorimuron + flumioxazin & $15.3+19.20$ & $900+9$ & 14.0 \\
Glyphosate + cloransulam & $15.3+19.20+23.97$ & $900+9+71$ & 14.1 \\
Glyphosate + flumioxazin & $15.3+23.97$ & $900+17.5$ & 15.5 \\
Glyphosate + glufosinate & $15.3+20.20$ & $900+71$ & 23.9 \\
Glyphosate + paraquat & $15.3+24.73$ & $900+500$ & 41.0 \\
Glyphosate + saflufenacil & $15.3+22.29$ & $900+1100$ & 14.4 \\
\hline
\end{tabular}

${ }^{\mathrm{z}}$ Abbreviations: EIQ, environmental impact quotient; EI, environmental impact.

Table 5. Percent control of multiple resistant (Group 2 and 9) common ragweed 4 WAA in the "burndown plus residual” study conducted in Ontario, Canada in 2013 and $2014^{\mathrm{z}}$.

\begin{tabular}{|c|c|c|c|c|}
\hline \multirow[t]{2}{*}{ Treatment } & \multirow{2}{*}{$\begin{array}{c}\text { Rate } \\
\text { (g a.i./a.e. ha }{ }^{-1} \text { ) }\end{array}$} & \multicolumn{3}{|c|}{ Percent control 4 WAA } \\
\hline & & S1 & S2 & S3, S4 \\
\hline Weedy control & & $0 \mathrm{~g}$ & $0 \mathrm{i}$ & $0 \mathrm{~g}$ \\
\hline Weed free control & & 100 a & $100 \mathrm{a}$ & 100 a \\
\hline Glyphosate & 900 & $13 \mathrm{f}$ & $11 \mathrm{~h}$ & $42 \mathrm{f}$ \\
\hline Glyphosate + chlorimuron & $900+9$ & 27 ef & $22 \mathrm{fg}$ & 60 e \\
\hline Glyphosate + chlorimuron + metribuzin & $900+9+412.5$ & 67 bc & 28 ef & 89 bc \\
\hline Glyphosate + clomazone & $900+846$ & 27 ef & $22 \mathrm{fg}$ & 58 e \\
\hline Glyphosate + cloransulam & $900+35$ & 33 e & $23 \mathrm{fg}$ & 58 e \\
\hline Glyphosate + flumetsulam & $900+70$ & 33 e & $47 \mathrm{~cd}$ & $58 \mathrm{e}$ \\
\hline Glyphosate + flumioxazin & $900+71.4$ & 27 ef & $60 \mathrm{bc}$ & 68 de \\
\hline Glyphosate + flumioxazin + chlorimuron & $900+71.4+9$ & 35 e & $43 \mathrm{~cd}$ & $71 \mathrm{~d}$ \\
\hline Glyphosate + flumioxazin/pyroxasulfone & $900+240$ & $53 \mathrm{~cd}$ & $74 \mathrm{ab}$ & 88 bc \\
\hline Glyphosate + imazethapyr & $900+100$ & $23 \mathrm{ef}$ & $15 \mathrm{gh}$ & 61 de \\
\hline Glyphosate + imazethapyr + metribuzin & $900+100+400$ & $52 \mathrm{~d}$ & 32 def & $85 \mathrm{c}$ \\
\hline Glyphosate + imazethapyr/saflufenacil & $900+100$ & $75 \mathrm{~b}$ & $64 \mathrm{bc}$ & $94.5 \mathrm{abc}$ \\
\hline Glyphosate + linuron & $900+2250$ & 99 a & $88 \mathrm{ab}$ & $98 \mathrm{ab}$ \\
\hline Glyphosate + metribuzin & $900+1120$ & 96 a & $86 \mathrm{ab}$ & $98 \mathrm{ab}$ \\
\hline Glyphosate + s-metolachlor/metribuzin & $900+1943$ & $57 \mathrm{~cd}$ & 36 de & 89 bc \\
\hline Glyphosate + saflufenacil/dimethenamid-p & $900+245$ & $80 \mathrm{~b}$ & $77 \mathrm{ab}$ & $95 \mathrm{ab}$ \\
\hline
\end{tabular}

${ }^{\mathrm{z}}$ Abbreviations: S1, S2, S3, S4, Windsor; WAA, weeks after application of herbicide. a - i means followed by the same letter are not significantly different according to Fisher's Protected LSD at $\mathrm{P}=0.05$.

the tankmixes evaluated in this study, with control ratings equivalent to glyphosate alone. Glyphosate plus chlorimuron provided $13 \%$ to $66 \%$ control. The addition of flumioxazin to glyphosate plus chlorimuron resulted in slightly higher control from $27 \%$ to $72 \%$. These results are in contrast to the results reported by Pollard [6] where greater than $80 \%$ control was achieved with a tankmix of glyphosate and chlorimuron. The difference in results between the two studies can be attributed to the fact that the Ontario biotype is resistant to both glyphosate and chlorimuron (unpublished data). Tank mixes of glyphosate plus cloransulam, flumioxazin, glufosinate or paraquat provided highly variable (i.e. $20 \%$ to $82 \%$ ) control of GR common ragweed. The only tankmix that provided control similar to the weed free control was glyphosate plus saflufenacil which provided $89 \%-90 \%$ 
Table 6. Percent control of multiple resistant (Group 2 and 9) common ragweed 8 WAA in the "burndown plus residual” study conducted in Ontario, Canada in 2013 and 2014 2 .

\begin{tabular}{|c|c|c|c|c|c|}
\hline \multirow[t]{2}{*}{ Treatment } & \multirow{2}{*}{$\begin{array}{c}\text { Rate } \\
\left(\text { (g a.i./a.e. } \text { ha }^{-1} \text { ) }\right.\end{array}$} & \multicolumn{4}{|c|}{ Percent control 8 WAA } \\
\hline & & S1 & S2 & S3 & S4 \\
\hline Weedy control & & $0 \mathrm{~h}$ & $0 \mathrm{~h}$ & $0 \mathrm{j}$ & $0 \mathrm{~d}$ \\
\hline Weed free control & & $100 \mathrm{a}$ & $100 \mathrm{a}$ & $100 \mathrm{a}$ & $100 \mathrm{a}$ \\
\hline Glyphosate & 900 & $17 \mathrm{~g}$ & $10 \mathrm{gh}$ & $40 \mathrm{i}$ & $43 \mathrm{c}$ \\
\hline Glyphosate + chlorimuron & $900+9$ & $20 \mathrm{fg}$ & $20 \mathrm{~g}$ & $57 \mathrm{~h}$ & $47 \mathrm{c}$ \\
\hline Glyphosate + chlorimuron + metribuzin & $900+9+412.5$ & $53 \mathrm{~cd}$ & 27 efg & 78 cde & $83 a b$ \\
\hline Glyphosate + clomazone & $900+846$ & $20 \mathrm{fg}$ & $18 \mathrm{~g}$ & $60 \mathrm{gh}$ & $47 \mathrm{c}$ \\
\hline Glyphosate + cloransulam & $900+35$ & 33 ef & 27 efg & $57 \mathrm{~h}$ & $40 \mathrm{c}$ \\
\hline Glyphosate + flumetsulam & $900+70$ & $20 \mathrm{fg}$ & 47 de & $57 \mathrm{~h}$ & $40 \mathrm{c}$ \\
\hline Glyphosate + flumioxazin & $900+71.4$ & $20 \mathrm{fg}$ & $56 \mathrm{~cd}$ & $67 \mathrm{fg}$ & $47 \mathrm{c}$ \\
\hline Glyphosate + flumioxazin + chlorimuron & $900+71.4+9$ & $20 \mathrm{fg}$ & $57 \mathrm{~cd}$ & 70 ef & $48 \mathrm{c}$ \\
\hline Glyphosate + flumioxazin/pyroxasulfone & $900+240$ & 40 de & $73 \mathrm{bc}$ & 76 de & $80 \mathrm{~b}$ \\
\hline Glyphosate + imazethapyr & $900+100$ & $23 \mathrm{fg}$ & $23 \mathrm{fg}$ & $57 \mathrm{~h}$ & $48 \mathrm{c}$ \\
\hline Glyphosate + imazethapyr + metribuzin & $900+100+400$ & 42 de & 47 de & 74 def & $50 \mathrm{c}$ \\
\hline Glyphosate + imazethapyr/saflufenacil & $900+100$ & $60 \mathrm{c}$ & $65 \mathrm{bcd}$ & $83 \mathrm{bcd}$ & $78 \mathrm{~b}$ \\
\hline Glyphosate + linuron & $900+2250$ & $80 \mathrm{~b}$ & $84 \mathrm{ab}$ & $91 \mathrm{~b}$ & $92 \mathrm{ab}$ \\
\hline Glyphosate + metribuzin & $900+1120$ & $82 \mathrm{~b}$ & $80 a b$ & 83 bcd & $95 \mathrm{ab}$ \\
\hline Glyphosate + s-metolachlor/metribuzin & $900+1943$ & 33 ef & 43 def & 75 def & $83 \mathrm{ab}$ \\
\hline Glyphosate + saflufenacil/dimethenamid-p & $900+245$ & $75 \mathrm{~b}$ & 74 bc & 85 bc & $79 \mathrm{~b}$ \\
\hline
\end{tabular}

${ }^{\mathrm{z}}$ Abbreviations: S1, S2, S3, S4, Windsor; WAA, weeks after application of herbicide. a - j means followed by the same letter are not significantly different according to Fisher's Protected LSD at $\mathrm{P}=0.05$.

Table 7. Multiple resistant (Group 2 and 9) common ragweed biomass 8 WAA and soybean yield in the "burndown plus residual” study conducted in Ontario, Canada in 2013 and $2014^{\mathrm{y}, \mathrm{z}}$.

\begin{tabular}{|c|c|c|c|c|}
\hline & & \multicolumn{2}{|c|}{ Weed biomass 8 WAA } & \multirow{2}{*}{$\begin{array}{c}\text { Soybean yield } \\
\text { S1, S3, S4 }\end{array}$} \\
\hline & Rate & S1, S2 & S3, S4 & \\
\hline Treatment & (g a.i./a.e. ha ${ }^{-1}$ ) & & & \\
\hline Weedy control & & 318 a & 169 a & 90 a \\
\hline Weed free control & & $0 \mathrm{~g}$ & $0 \mathrm{e}$ & $0 \mathrm{j}$ \\
\hline Glyphosate & 900 & $175 \mathrm{ab}$ & $144 \mathrm{ab}$ & $79 \mathrm{ab}$ \\
\hline Glyphosate + chlorimuron & $900+9$ & $169 \mathrm{abc}$ & 162 a & $81 \mathrm{ab}$ \\
\hline Glyphosate + chlorimuron + metribuzin & $900+9+412.5$ & 135 bc & 47 de & 45 efgh \\
\hline Glyphosate + clomazone & $900+846$ & $140 \mathrm{bc}$ & $129 \mathrm{abc}$ & 67 bcde \\
\hline Glyphosate + cloransulam & $900+35$ & $150 \mathrm{bc}$ & $149 \mathrm{ab}$ & 70 abcd \\
\hline Glyphosate + flumetsulam & $900+70$ & 112 bc & $132 \mathrm{abc}$ & $71 \mathrm{abc}$ \\
\hline Glyphosate + flumioxazin & $900+71.4$ & 89 bcd & $151 \mathrm{ab}$ & 64 bcde \\
\hline Glyphosate + flumioxazin + chlorimuron & $900+71.4+9$ & 109 bc & $135 \mathrm{ab}$ & 62 bcdef \\
\hline Glyphosate + flumioxazin/pyroxasulfone & $900+240$ & 77 cdef & 45 de & 51 cdefg \\
\hline Glyphosate + imazethapyr & $900+100$ & $173 \mathrm{ab}$ & $130 \mathrm{abc}$ & 63 bcde \\
\hline Glyphosate + imazethapyr + metribuzin & $900+100+400$ & 78 bcde & 81 bcd & 48 defgh \\
\hline Glyphosate + imazethapyr/saflufenacil & $900+100$ & 57 cdef & 35 de & 39 ghi \\
\hline Glyphosate + linuron & $900+2250$ & $10 \mathrm{fg}$ & 11 de & 27 hi \\
\hline Glyphosate + metribuzin & $900+1120$ & 14 efg & 7 de & $20 \mathrm{ij}$ \\
\hline Glyphosate + s-metolachlor/metribuzin & $900+1943$ & 133 bc & $57 \mathrm{~cd}$ & 54 cdefg \\
\hline Glyphosate + saflufenacil/dimethenamid-p & $900+245$ & 16 def & $27 \mathrm{de}$ & 40 fghi \\
\hline
\end{tabular}

${ }^{\mathrm{z}}$ Abbreviations: S1, S2, S3, S4, Windsor; WAA, weeks after application of herbicide. ${ }^{\mathrm{y}}$ Compared to the weed free control. a - $\mathrm{g}$ means followed by the same letter are not significantly different according to Fisher's Protected LSD at P $=0.05$. 
Table 8. Environmental impact of herbicides used in the "burndown plus residual" study conducted in Ontario, Canada in 2013 and $2014^{\mathrm{z}}$.

\begin{tabular}{cccc}
\hline Active ingredient & Individual EIQ values & Product rate & EI \\
\hline Glyphosate & & (g a.i/a.e. ha ${ }^{-1}$ ) & 13.8 \\
Glyphosate + chlorimuron & 15.3 & 900 & 14.0 \\
Glyphosate + chlorimuron + metribuzin & $15.3+19.2$ & $900+9$ & 25.7 \\
Glyphosate + clomazone & $15.3+19.20+28.37$ & $900+9+412.5$ & 30.4 \\
Glyphosate + cloransulam & $15.3+19.63$ & $900+846$ & 14.1 \\
Glyphosate + flumetsulam & $15.3+15.33$ & $900+17.5$ & 14.9 \\
Glyphosate + flumioxazin & $15.3+15.61$ & $900+70$ & 15.5 \\
Glyphosate + flumioxazin + chlorimuron & $15.3+23.97$ & $900+71.4$ & 15.7 \\
Glyphosate + flumioxazin/pyroxasulfone & $15.3+23.97 / 12.33$ & $900+71.4+9$ & 15.8 \\
Glyphosate + imazethapyr & $15.3+19.57$ & $900+240$ & 27.1 \\
Glyphosate + imazethapyr + metribuzin & $15.3+19.57+28.37$ & $900+100+400$ & 14.2 \\
Glyphosate + imazethapyr/saflufenacil & $15.3+19.57 / 22.29$ & $900+100$ & 57.3 \\
Glyphosate + linuron & $15.3+19.32$ & $900+2250$ & 45.6 \\
Glyphosate + metribuzin & $15.3+28.37$ & $900+1120$ & 18.2 \\
Glyphosate + s-metholachlor/metribuzin & $15.3+22.00 / 28.37$ & $900+1943$ & 17.0 \\
Glyphosate + saflufenacil/dimethenamid-p & $15.3+22.29 / 12.02$ & $900+245$ & \\
\hline
\end{tabular}

${ }^{\mathrm{z}}$ Abbreviations: EIQ, environmental impact quotient; EI, environmental impact.

control at S2, S3 and S4, but only $80 \%$ control at S1, which was not equivalent to the weed free control. Therefore, control with glyphosate plus saflufenacil was not consistent between years and sites. This is similar to results found by Byker et al. [13] where a tankmix of glyphosate plus saflufenacil provided the best control of GR Canada fleabane, but results were not consistent among sites.

At 8 WAA, S1 and S2 data could be combined and S3 and S4 control data could be combined. None of the herbicides provided control equivalent to the weed free control (Table 2). Glyphosate plus saflufenacil provided the highest control at 8 WAA, however, the control was only 69\% and 76\% at S1, S2 and S3, S4, respectively. Glyphosate tankmixed with carfentrazone, chlorimuron and cloransulam provided control equivalent to glyphosate alone at $20 \%$ to $45 \%, 18 \%$ to $52 \%$, and $17 \%$ to $53 \%$ respectively. The decline in control from 4 to 8 WAA is attributed to the long emergence pattern of common ragweed, where new plants were still emerging in July and August (personal observation). These later emerging common ragweed seedlings were not controlled by the herbicides with relatively short residual activity that were applied in the latter half of May.

Weed biomass data for S1 and S2 could be combined while S3 and S4 were analyzed separately (Table 3). The results of S4 were not consistent with the results found at the other sites which may be due to the lower density of common ragweed at S4 (Table 1) and the lower proportion of resistant common ragweed biotypes at S4 (unpublished data). At S1, S2 and S3, weed biomass was reduced 27\% to 100\% compared to the untreated control. At S1, S2; S3 and S4, the addition of saflufenacil to glyphosate resulted in a 79\%, $41 \%$ and $100 \%$ reduction in weed biomass, respectively. Glyphosate plus carfentrazone resulted in the highest weed biomass, similar to the untreated control at S1, S2. Glyphosate plus 2,4-D, amitrole, glufosinate, paraquat or saflufenacil reduced GR common ragweed biomass by more than 89\%, 46\%, 56\% and 41\%, respectively.

GR common ragweed interference resulted in a soybean yield reduction of $73 \%$ at S1, S3 (Table 3). Treatments of glyphosate, glyphosate plus 2,4-D, glyphosate plus carfentrazone, glyphosate plus chlorimuron, glyphosate plus chlorimuron plus flumioxazin, glyphosate plus cloransulam and glyphosate plus flumioxazin resulted in soybean reductions equal to the weedy control at S1, S3. All of the herbicide treatments resulted in a soybean loss. Glyphosate plus saflufenacil had the lowest yield loss of $24 \%$. The same results were not seen at S2, likely due to the lower common ragweed interference in the experiment (results not shown).

The most effective tankmix in the enhanced burndown study-glyphosate plus saflufenacil—has a relatively low EI when compared with many tank mix treatments in this study. Glyphosate plus saflufenacil had an EI of 14.4 compared to glyphosate at 13.8 (Table 4). The only two tankmixes with a lower EI were glyphosate plus 
either chlorimuron or cloransulam which have EIs of 14.0 and 14.1, respectively. Glyphosate plus amitrole or paraquat had the highest EIs of 77.4 and 41.0, respectively. The level of efficacy of glyphosate plus saflufenacil and the relatively low EI of this tank mix make it a desirable option for control of GR common ragweed.

\subsection{Burndown plus Residual}

At 4 WAA, glyphosate plus linuron or metribuzin provided $88 \%$ to $99 \%$ and $86 \%$ to $98 \%$ control of GR common ragweed, respectively (Table 5). These two tankmixes were the most effective of the herbicides evaluated, and the control was equivalent to the weed free control. The control of GR common ragweed with glyphosate plus metribuzin is similar to the control of GR Canada fleabane reported by Byker et al. [13] where glyphosate plus metribuzin provided 98\% to $100 \%$ control of GR Canada fleabane in Ontario. Interestingly, Vink et al. [14] reported $95 \%$ to $98 \%$ control of GR giant ragweed 4 WAA with glyphosate plus linuron. Glyphosate plus saflufenacil/dimethenamid-p provided 77\% to 95\% GR common ragweed control at S2 and S3, S4 which was equivalent to the weed free control, however, at S1 control was less than the weed free control. Glyphosate plus flumioxazin/pyroxasulfone provided control similar to the weed free control at S2, but not at the other three sites. Glyphosate plus imazethapyr/saflufenacil provided control similar to the weed free control at two of the four sites (S3, S4), however, control was 75\% and 64\% at S1 and S2, respectively. All other herbicides in this study did not provide control equivalent to the weed free control. The control of GR common ragweed with glyphosate plus chlorimuron plus metribuzin was less than glyphosate plus metribuzin at all sites which can be attributed to the lower rate of metribuzin. Similarly, the addition of metribuzin to glyphosate plus imazethapyr, although it did result in improved control, was not equivalent to glyphosate plus metribuzin, which can be attributed to the lower rate of metribuzin used. The improved herbicide efficacy at S3, S4 may be due to the lower proportion of resistant biotypes at S4 (unpublished data). The results of this study are in contrast to Pollard [6] who found that glyphosate plus chlorimuron or imazethapyr provided greater than $80 \%$ control, while in this study both tankmixes provided less than $61 \%$ control. This difference is thought to be due to multiple resistant (Group 2 and 9) common ragweed at all sites in this study (unpublished data).

At 8 WAA, glyphosate plus linuron or metribuzin provided $80 \%$ to $92 \%$ and $80 \%$ to $95 \%$ GR common ragweed control, respectively (Table 6) which was equivalent to the weed free control at 2 of the 4 sites. Glyphosate plus linuron or metribuzin provided control similar to the weed free control at S2 and S4, but not at S1and S3. Glyphosate plus a Group 2 herbicide (chlorimuron, cloransulam, flumetsulam or imazethapyr) provided control similar to glyphosate alone at two of the four sites which is attributed the multiple resistant biotype found at these sites.

At 8 WAA, glyphosate plus linuron or metribuzin reduced GR common ragweed biomass $94 \%$ to $97 \%$ and $96 \%$, respectively, which was equivalent to the weed free control (Table 7). At S3 and S4, glyphosate plus chlorimuron plus metribuzin, flumioxazin/pyroxasulfone, imazethapyr/saflufenacil or saflufenacil/dimethenamid-p reduced common ragweed biomass by $72 \%, 73 \%, 79 \%$ and $84 \%$, respectively which was equivalent to the weed free control. Glyphosate plus chlorimuron or imazethapyr both resulted in GR common ragweed biomass that was equivalent to the untreated control. The other Group 2 herbicides (cloransulam and flumetsulam) had biomass reductions equivalent to the untreated control at S3, S4 but similar to glyphosate alone across all sites.

GR common ragweed interference resulted in soybean yield reductions of $90 \%$ in the weedy control compared to the weed free control (Table 7). GR common ragweed interference following the application of glyphosate plus metribuzin at S1, S3, S4, resulted in soybean yield loss of $20 \%$ which was equivalent to the weed free control. Treatments of glyphosate, glyphosate plus chlorimuron, glyphosate plus cloransulam and glyphosate plus flumetsulam had yield reductions similar to the weedy control.

Glyphosate plus linuron or metribuzin provided the best control of GR common ragweed, unfortunately, these tankmixes also had the highest EI of the herbicides in this study at 57.3 and 45.6 respectively (Table 8). The treatment with the lowest EI is glyphosate at 13.8, followed by glyphosate plus chlorimuron or cloransulam at 14 and 14.1 respectively. However, neither of these herbicides provided adequate control of the multiple resistant common ragweed. The other herbicides that had weed biomass similar to the weed free control at half the sites, chlorimuron plus metribuzin, imazethapyr/saflufenacil and saflufenacil/dimethenamid-p, have an EI of 25.7, 14.2 and 23.4, respectively. Therefore, from an environmental standpoint, the tankmix of glyphosate plus imazethapyr/saflufenacil would be the best option, however, would only reduce GR common ragweed biomass by $79 \%$ to $82 \%$ compared to the $94 \%$ to $97 \%$ that could be achieved with linuron or metribuzin. 


\section{Conclusion}

The most efficacious herbicide treatments for the control of GR common ragweed are glyphosate tankmixed with either linuron or metribuzin, which provided the most consistent full season control across sites and years. The tankmixes of glyphosate plus a Group 2 herbicide did not provide adequate control and in some cases resulted in control equivalent to glyphosate alone. This result indicates that the common ragweed biotype at these sites is multiple resistant and another mode of action is required to control this biotype. In addition, because of the long emergence pattern of common ragweed in Ontario, a herbicide with residual activity is required for full season control. Because of the few options available for the control of multiple resistant common ragweed, it is important that it is controlled to decrease spread to adjacent fields. Future research should focus on two-pass weed control programs of a preplant herbicide followed by a postemergence herbicide for full season control of this competitive weed in soybean.

\section{References}

[1] Ontario Ministry of Agriculture, Food, and Rural Affairs (2014) Ontario Weeds: Giant Ragweed. http://www.omafra.gov.on.ca/english/crops/facts/ontweeds/giant_ragweed.htm

[2] Frick, B. and Thomas, A.G. (1992) Weed Surveys in Different Tillage Systems in Southwestern Ontario Field Crops. Canadian Journal of Plant Science, 72, 1337-1347. http://dx.doi.org/10.4141/cjps92-166

[3] Coble, H.D., Williams, F.M. and Ritter, R.L. (1981) Common Ragweed (Ambrosia artemisiifolia) Interference in Soybeans (Glycine max). Weed Science, 29, 339-342.

[4] Alex, J.F. and Switzer, C.M. (1975) Ontario Weeds. Publication 505. Ministry of Agriculture, Food and Rural Affairs, Ontario, Canada. Queen's Printer for Ontario, Toronto, 200 p.

[5] Cowbrough, M.J. (2006) Noxious Weed Profile-Ragweed spp. http://www.omafra.gov.on.ca/english/crops/facts/info_ragweed.htm

[6] Pollard, J.M. (2007) Identification and Characterization of Glyphosate-Resistant Common Ragweed (Ambrosia artemisiifolia L.). M.Sc. Dissertation, University of Missouri, Columbia.

[7] Heap, I. (2014) The International Survey of Herbicide Resistant Weeds. www.weedscience.com

[8] Kovach, J., Petzoldt, J., Degnil, J. and Tette, J. (2004) A Method to Measure the Environmental Impact of Pesticides. New York's Food and Life Sciences Bulletin, 139, 139-146.

[9] Bues, R., Dadomo, M., Lyannaz, J.P., Di Lucca, G., Macua Gonzalez, J.I., Prieto Losada, H. and Dumas, Y. (2003) Evaluation of the Environmental Impact of the Pesticides Applied in Processing Tomato Cropping. Acta Horticulturae, 61, 255-258.

[10] Fernandez-Cornejo, J. (1998) Environmental and Economic Consequences Adoption: IPM in Viticulture. Agricultural Economics, 18, 145-155. http://dx.doi.org/10.1016/S0169-5150(97)00054-6

[11] Kovach, J., Petzoldt, J., Degnil, J. and Tette, J. (2012) Table 2. List of Pesticides. http://www.nysipm.cornell.edu/publications/eiq/equation.asp

[12] Ziegler, C.R., Donahue, D.W., Drummond, F.A. and Smith, S.N. (2002) The Ecological Economics of Insecticide Use Associated with the Maine Potato Industry Based on a Producer Survey. American Journal of Alternative Agriculture, 17, 159-166. http://dx.doi.org/10.1079/AJAA2002159

[13] Byker, H.P., Soltani, N., Robinson, D., Tardif, F., Lawton, M. and Sikkema, P.H. (2013) Occurrence of Glyphosate and Cloransulam Resistant Canada Fleabane (Conyza canadensis L. Cronq.) in Ontario. Canadian Journal of Plant Science, 93, 851-855. http://dx.doi.org/10.4141/cjps2013-039

[14] Vink, J.P., et al. (2012) Glyphosate-Resistant Giant Ragweed (Ambrosia trifida L.) Control with Preplant Herbicides in Soybean [Glycine max (L.) Merr.]. Canadian Journal of Plant Science, 92, 913-922.

http://dx.doi.org/10.4141/cjps2012-025 
Scientific Research Publishing (SCIRP) is one of the largest Open Access journal publishers. It is currently publishing more than 200 open access, online, peer-reviewed journals covering a wide range of academic disciplines. SCIRP serves the worldwide academic communities and contributes to the progress and application of science with its publication.

Other selected journals from SCIRP are listed as below. Submit your manuscript to us via either submit@scirp.org or Online Submission Portal.
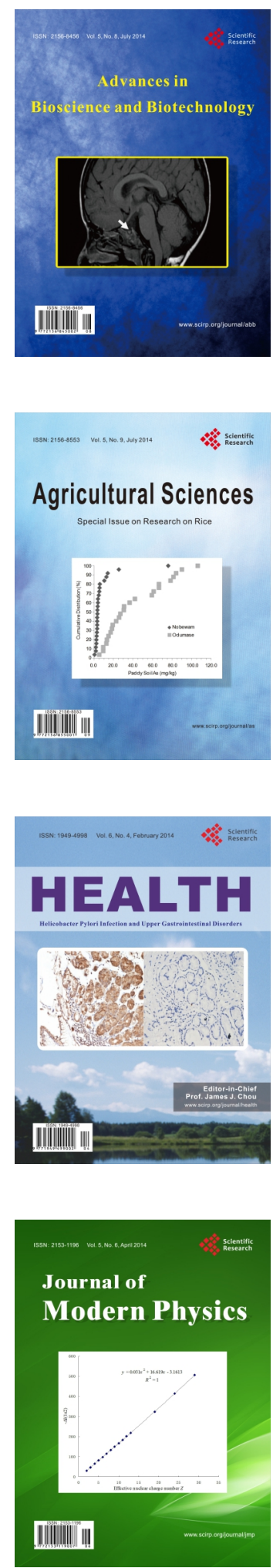
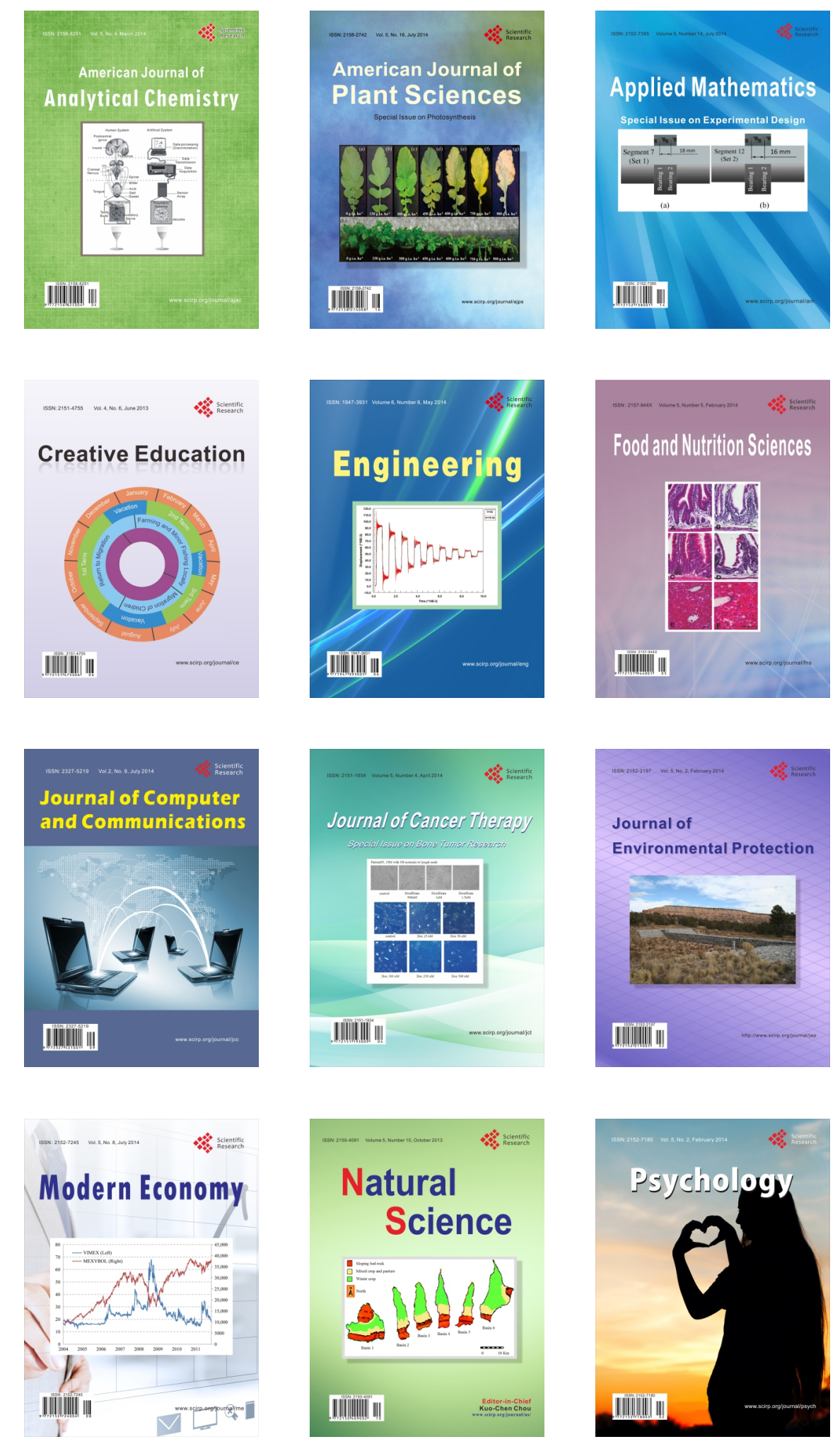